\title{
Monitoring and guidance of high intensity focused ultrasound exposures in real time using acousto-optic imaging: feasibility and demonstration ex vivo
}

\author{
Puxiang Lai, James R. McLaughlan, ${ }^{*}$ Andrew B. Draudt, ${ }^{*}$ Todd W. Murray, ${ }^{\dagger}$ \\ Robin O. Cleveland, and Ronald A. Roy \\ * Department of Mechanical Engineering, Boston University, \\ 110 Cummington Street, Boston, MA 02215 USA \\ ${ }^{\dagger}$ Department of Mechanical Engineering, University of Colorado at Boulder \\ 427 UCB, Engineering Center, Boulder CO 80309 USA
}

\begin{abstract}
High intensity focused ultrasound (HIFU) is a powerful noninvasive tool for targeted tissue ablation. Monitoring of the treatment process and efficacy in real time, however, remains challenging. The tissue necrosis during the HIFU exposure leads to changes in optical absorption and scattering coefficients. In this paper, we explore the use of acousto-optic imaging (AOI), a hybrid technique that combines ultrasound with diffuse light to obtain deep-tissue optical contrast at ultrasound resolution, to sense the changes in optical contrast at depth in tissue associated with the onset formation and development of the necrosed tissue region. In this technique, the tissue to be treated is illuminated with near-infrared light and a continuous, amplitude-modulated focused ultrasound beam is used to induce thermal tissue necrosis as well as the acousto-optic (AO) interaction. The AOI signal is detected via a photorefractive crystal (PRC)-based interferometer, and then fed into a lock-in amplifier tuned to the ultrasound modulation frequency. As a thermal lesion forms in the ultrasound focal zone, which is also the zone of AO interaction, the AOI signal diminishes in amplitude owing to enhanced optical attenuation. It is further shown that the reduction of the AOI signal is correlated with the volume of ensuing lesion. Therefore, the evolution of AOI signal as a function of time provides a means for continuous monitoring of HIFU treatment process as well as exposure guidance.
\end{abstract}

Keywords: high intensity focused ultrasound, treatment monitoring and guidance, acousto-optic imaging, ultrasound-modulated optical tomography, photorefractive crystal, lesion volume

\section{INTRODUCTION}

High intensity focused ultrasound (HIFU) is a powerful and noninvasive technique that is capable of delivering high intensity acoustic energy to a selected region at depth in tissue, causing localized thermal coagulative necrosis while

Further author information: Puxiang Lai, lpx@bu.edu, 1-617-353 4854

Prof. Ronald A. Roy, ronory@bu.edu, 1-617-353 4846

Photons Plus Ultrasound: Imaging and Sensing 2010, edited by Alexander A. Oraevsky, Lihong V. Wang, Proc. of SPIE Vol. $7564,75642 B \cdot$ - C 2010 SPIE · CCC code: 1605-7422/10/\$18 - doi: 10.1117/12.842358 
minimizing or avoiding adverse affects in the surrounding tissue ${ }^{1}$. The necrosed region produced by HIFU exposure is generally referred to as a lesion. HIFU was first proposed for the remedy of Parkinson's disease more than 50 years ago ${ }^{2}$, and it has since been successfully applied for the clinical ablation of solid tumors in soft tissues like liver ${ }^{3}$, kidney ${ }^{4}$, prostate $^{5}$, uterine fibroids ${ }^{6}$, breast ${ }^{7}$, sarcoma ${ }^{8}$ and pancreas ${ }^{9}$. Typically, the amount of acoustic energy absorbed by the tissue and the resulting temperature rise is estimated based on the assumption that the attenuation of sound in tissue between the transducer and the focal region is linear ${ }^{10}$, which, unfortunately, is not always the case. It is complicated by the variation in the physical properties and functional status of the intermediate tissue. The sound absorption can also have time and temperature dependence, leading to uncertainty in the extent of the necrosed region, and ultimately limiting the reliability of treatment planning and efficacy evaluation ${ }^{11}$. Therefore there is need for real-time monitoring techniques to ensure that lesions of a desired volume are created and damage to the surrounding tissue is minimized.

Several non-invasive modalities have been developed for HIFU treatment monitoring and guidance, among which magnetic resonance imaging (MRI) and diagnostic ultrasound have received the most attention and been implemented clinically. Based on changes in proton resonant frequency shift with temperature, MRI is able to differentiate different types of tissue and map the temperature field from the HIFU exposure with promising spatial and temperature resolution. It is regarded as the current 'gold' standard in HIFU treatment guidance ${ }^{11,12}$. However, this imaging technique is limited by the effective frame rate, system cost, and the requirement that the sample be exposed to magnetic fields. Diagnostic ultrasound can detect lesions based on mechanical/acoustic properties variations that are produced as a lesion is formed. It offers real-time imaging with reasonable spatial resolution and is relatively inexpensive and portable. However, the contrast between the lesion and normal tissue is small and somewhat difficult to detect using a standard B-mode scan. Typically, the identifiable feature resulting from the HIFU exposures is hyperechogenicity in the B-mode images, which is thought to be related to boiling during the exposure ${ }^{13}$. The occurrence of boiling can indicate overtreatment in a particular region, and boiling during HIFU treatment may irregularly distort the location, shape and size of the necrotic region.

Tissue necrosis is accompanied by increases in optical absorption and scattering coefficients, ${ }^{14-19}$ opening up the possibility of monitoring HIFU treatment through the detection of optical contrast in the focal zone. Pure optical imaging is not suitable for this application due to the strong optical scattering exhibited by biological tissue, limiting the resolution that can be achieved at depth. Optoacoustic tomography (OAT) $)^{18,20}$ has been used to detect ${ }^{18}$ or visualize ${ }^{20}$ the necrosed region in ex vivo soft tissue based on optical contrast between normal and necrosed regions. Thermoacoustic tomography (TAT) ${ }^{21}$ has also been used to image thermal lesions. These studies were aimed at the sensing and imaging the lesions, rather than real-time monitoring of lesion formation.

In this paper, we demonstrate that acousto-optic imaging (AOI) can be used to sense the dynamic changes in optical contrast at depth in tissue associated with the formation of necrosed tissue region during HIFU exposure. In AOI, an ultrasound beam is applied to affect the propagation of multiply scattered light in tissue within the acousto-optic (AO) interaction region ${ }^{22-24}$, defined by where light and sound co-exist. The sound imparts a net phase or frequency shift onto the light that traverses the ultrasound field through ultrasound induced displacement of optical scatterers and pressure induced changes in refractive index ${ }^{25,26}$. The intensity of the phase shifted light detected external to the sample and the magnitude of the phase shift imparted on the light are both related to the optical properties of the AO interaction region. 
The AO signal can thus be used to monitor optical property changes in the interaction region, with the spatial resolution dictated by the spatial extent of the ultrasound beam.

\section{METHODOLOGY AND MATERIALS}

\subsection{Experimental Setup}

The experimental setup is illustrated in Fig. 1. The setup can be roughly divided into four subsystems: a) a HIFU system to provide both the local heating and ultrasound modulation in the sample (marked with orange lines), b) a PRC-based AOI system operating at $1064 \mathrm{~nm}$ optical wavelength (marked with red lines), c) a passive cavitation detection (PCD) system to monitor the broadband emissions from the inertial cavitation (marked with green lines), and d) a Terason diagnostic ultrasound imaging system to determine whether or not boiling is generated during the exposures (with the ultrasound probe labeled with "TUP”).

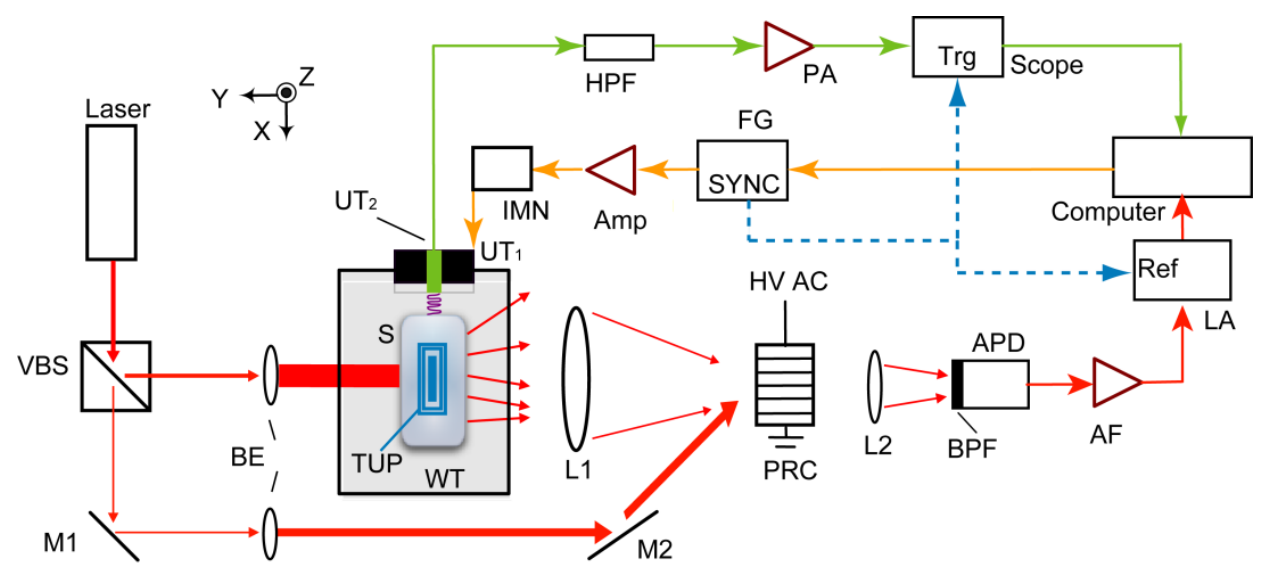

Fig. 1. System apparatus: $X Y Z-$ system coordinate ( $\mathrm{Z}$ is the vertical axis); VBS- variable beam splitter; $\mathrm{BE}_{1}, \mathrm{BE}_{2}-\mathrm{beam}$ expander; $\mathrm{M}_{1}$, $\mathrm{M}_{2}$ - mirror; TUP- Terason Ultrasound System probe (its acoustic axis along the Z axis); WT- tank filled with degassed water; Sdegassed chicken breast sample; $\mathrm{UT}_{1}-\mathrm{HIFU}$ transducer; $\mathrm{UT}_{2}-$ ultrasound transducer for passive-cavitation detection (PCD); $\mathrm{L}_{1}, \mathrm{~L}_{2}-$ lens; HV AC- high voltage AC electrical field; PRC- photorefractive crystal; BPF- band-pass filter; APD- avalanche photodiode; AFactive filter; LA- lock-in amplifier; FG- function generator; Amp- power amplifier; IMN- impedance matching network; PApreamplifier; HPF- high-pass filter; Scope- Oscilloscope; SYNC- synchronizing output; Trg- trigger signal; Ref- reference signal.

In previous experimental AOI experimental systems, the AO interaction was driven by continuous wave ultrasound ${ }^{24}$, millisecond-duration ultrasound bursts with low duty cycle ${ }^{27}$ or short ultrasound pulses ${ }^{28}$. In the present experiment, the AO response is driven by the HIFU field, a $1.1 \mathrm{MHz}$ sinusoidal waveform that is amplitude modulated at repetition rate of $50 \mathrm{~Hz}$ with $50 \%$ duty cycle (Fig. 2(a)). The HIFU field thus serves to generate both the AO response and the local heating in tissue during the exposure. The HIFU transducer (H102, Sonic Concepts, WA, USA) has a $10.5 \times 1.6 \times 1.6$ $\mathrm{mm}^{3}$ focal region along the XYZ axes. Prior to measurements, the HIFU axis was aligned perpendicular to the incident optical beam, with the focus aligned to the center of the beam. A function generator provides the modulated signal used to drive the HIFU transducer, a $50 \mathrm{~Hz}$ reference signal to the lock-in amplifier, and a trigger signal for data acquisition on the oscilloscope 


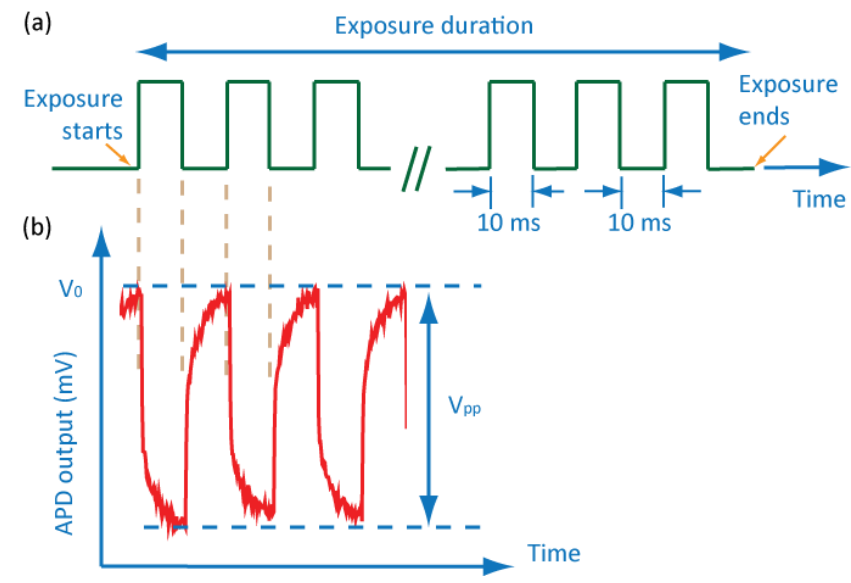

Fig. 2 (a) The amplitude modulated sinusoidal waves with central frequency of $1.1 \mathrm{MHz}$ during the HIFU exposure. The modulation frequency is $50 \mathrm{~Hz}$ and the duty cycle is $50 \%$. (b) An example of AOI waveform before being sent into the lock-in amplifier, which was generated by the HIFU field shown in (a) under a relatively low focal pressure (1 MPa peak positive in water) in ex vivo chicken breast tissue. It was coherently averaged over 1,000 sweeps (10 ms bursts), taking more than $20 \mathrm{~s}$.

The PRC-based AOI system is similar to that discussed in the literature ${ }^{29,30}$. An important distinction in the present case, however, is that the interferometer output is fed directly into a lock-in amplifier (SR830, Stanford Research System, CA, USA). The time domain signal detected using the HIFU transducer operating at $50 \%$ duty cycle at a repetition rate of $50 \mathrm{~Hz}$ is shown in Fig. 2(b), where it is evident that much of the signal energy is in the $50 \mathrm{~Hz}$ frequency range. Using lock-in detection (30 ms time constant and $12 \mathrm{~dB} /$ octave roll off), the bandwidth of the system can be greatly reduced, leading to significant improvement in signal-to-noise ratio (SNR) and opening up the possibility of real-time detection of the HIFU induced changes in sample properties. Note that a low ultrasound drive pressure was used to generate the AO response shown in Fig. 2(b). A decrease in the peak-to-peak AOI signal is observed at higher drive pressures as a thermal lesion is formed. Using lock-in detection, the magnitude output from the lock-in $\mathrm{S}(\mathrm{t})$, giving the RMS value of the APD output at $50 \mathrm{~Hz}$, is sampled by a computer at a rate of $7 \mathrm{~Hz}$ and displayed as a function of HIFU exposure time. The absolute value AOI amplitude is affected by a number of parameters outside of the local optical properties ${ }^{29,30}$. In order to compensate for this, we present a normalized AOI response $S_{n}(t)$, where $S(t)$ is divided by the mean magnitude of the signal during the initial part of the HIFU exposure $\mathrm{S}_{0}$, prior to any lesion formation. In practice, the time window from 2-5 $\mathrm{s}$ is chosen to calculate $\mathrm{S}_{0}$ based our experiences given focal pressures less than $10 \mathrm{MPa}$ in the experiment sample used in this study. The change of AOI amplitude during the exposure is given as

$$
\Delta S(t)=\left|S_{n}(t)-1\right| \times 100 \%=\left|S(t) / S_{0}-1\right| \times 100 \% .
$$

The passive cavitation detector (PCD) is a small-aperture, single-element transducer ( $\mathrm{UT}_{2}$ in Fig. 1) (7.5 MHz central frequency, Model 140-710-S, Olympus, MA, USA) that is installed in the center hole of the HIFU transducer and aligned concentrically and coaxially with respect to the HIFU focal peak. During the exposure, this transducer functions passively, receiving acoustic signals originating from its focal region. The transducer output is an indication of broadband acoustic emissions due to the inertial cavitation, registered as a function of time ${ }^{31,32}$. A Terason 2000 (Terason Tech, MA, USA) is used as the diagnostic ultrasound imaging system. It has a $7.5 \mathrm{MHz}$ central frequency, 
128-element linear probe (TUP in Fig. 1), B-mode images are acquired immediately before and after each HIFU exposure. The changes in echogenicity in the focal region indicate the presence of boiling during the exposure.

\subsection{Materials and exposure parameters}

Ex vivo store bought chicken breast tissue was used as the target material in this study. Samples were cut with dimensions $50 \times 15 \square 30 \times 50 \mathrm{~mm}^{3}$ along the $\mathrm{XYZ}$ axes. The samples were degassed for $3 \mathrm{hr}$ before usage, and mounted in the sample holder in the tank filled with freshly degassed (for $1 \mathrm{~h}$ ) water for the experiment. Each sample was used for 2 or 3 exposures in this study at different locations in the $\mathrm{Z}$ direction. The HIFU exposure parameters are shown in Table 1. For all exposures, the acoustic focus was approximately $16 \mathrm{~mm}$ into the tissue along the $\mathrm{X}$ axis (HIFU axis). The water temperature in the tank during each experiment was $21 \pm 2^{\circ} \mathrm{C}$.

Table 1 HIFU exposure parameters

\begin{tabular}{ccccc} 
& Group 1 & Group 2 & Group 3 & Group 4 \\
\hline "Focal pressure (MPa) & 8 & $6 \sim 10$ & 10 & 10 \\
Duration (s) & 40 & $5 \sim 60$ & ${ }^{*}$ TBD & $25 \sim 75$ \\
Number of exposures & 72 & 87 & 157 & 189 \\
\hline
\end{tabular}

* Peak positive focal pressure measured in water

₹ To be determined by the AOI response during the exposure

\subsection{Lesion volume characterization}
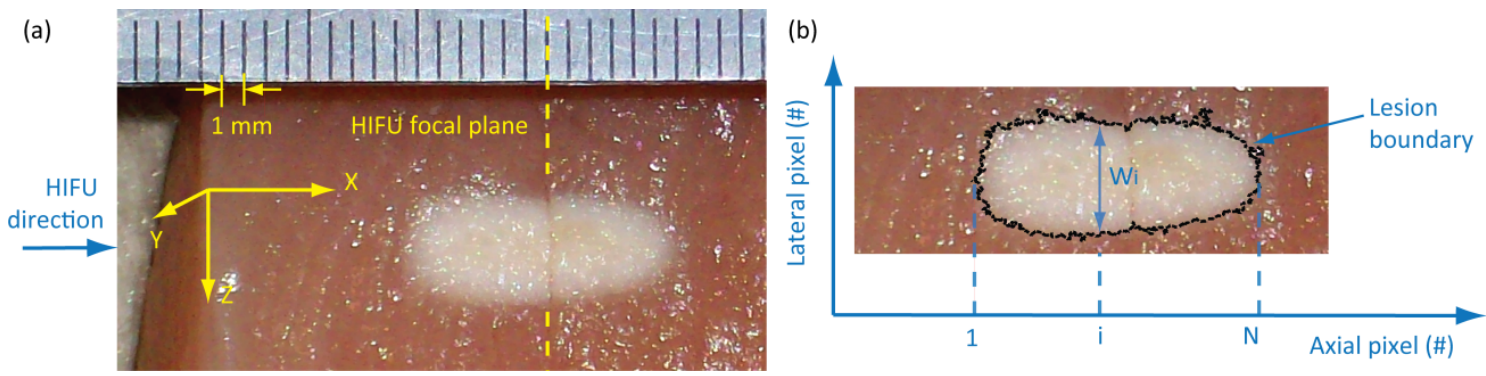

Fig. 3 (a) A cross-section photograph of a lesion produced in ex vivo chicken breast tissue sample by a $10 \mathrm{MPa}, 50 \mathrm{~s}$ exposure. The HIFU direction was from left to right with its focus $16 \mathrm{~mm}$ into the tissue. (b) The Matlab processed lesion with lesion boundary identified.

After exposure, each tissue sample was removed from the sample holder and sliced first along the HIFU focal plane (as shown in Fig. 3 (a)) and then along the XZ plane to obtain the lesion central cross-section. An example of a typical lesion photograph is shown in Fig. 3(a). The boundary of the lesion extent is identified based on an isointensity contour threshold method using Matlab software. The boundary of one lesion found using this approach is shown in Fig. 3(b). The volume of the lesion is estimated through:

$$
L V=\sum_{i=1}^{N}\left[\pi\left(\frac{W_{i}}{2}\right)^{2} /\left(C_{p}\right)^{3}\right]
$$

where $\mathrm{N}$ is the number of pixels along the length of the lesion, $\mathrm{W}_{\mathrm{i}}$ is the lesion width in the units of pixels at each axial position, and $\mathrm{C}_{\mathrm{p}}$ is the pixel calibration factor defined by the number of pixels per $\mathrm{mm}$ distance on the photograph. By 
using Eq. 2, it is assumed that the lesion is axis-symmetric on the plane perpendicular to the HIFU axis. This assumption, through our experiences, is reasonable for most of the lesions generated in tissue samples without major structural features, such as big blood vessels, and no inertial cavitation or boiling was generated during the exposures.

\section{RESULTS AND DISCUSSION}

Multiple exposures were performed in the prepared ex vivo chicken breast tissues with either fixed (Group 1, Table 1) or varied (Group 2, Table 1) pressures and durations. For each exposure, $\Delta S$ is calculated and recorded in parallel with the corresponding post-estimated lesion volume, the PCD supervising signals, and B-mode ultrasound images.

Fig. 4 (a)-(e) shows a typical result from a $10 \mathrm{MPa}, 40$ second exposure in the sample. In Fig. 4(a), the AOI amplitude S is relatively constant between $2 \sim 5 \mathrm{~s}$, having a mean value of 47.45 and a standard deviation of 1.30 . After $13 \mathrm{~s}$, the signal begins to decrease to a minimum value of 19.51 at $40 \mathrm{~s}$, when the exposure is turned off. The reduction of $\mathrm{S}$ is associated with the increased optical absorption and scattering coefficients accompanying the formation and development of the lesion in the AO interaction region. The PCD signal shown in Fig. 4 (b) suggests that no inertial cavitation is generated during the exposure. In Fig. 4 (c) and (d), there is no change in the echogenicity observed in the HIFU focus, indicating boiling does not occur. Fig. 4 (e) shows the photograph of the lesion generated under this exposure. It is important to note that the generation and presence of a lesion cannot be detected by B-mode ultrasound imaging in this case, but it is clearly evident in the $\mathrm{AO}$ response.
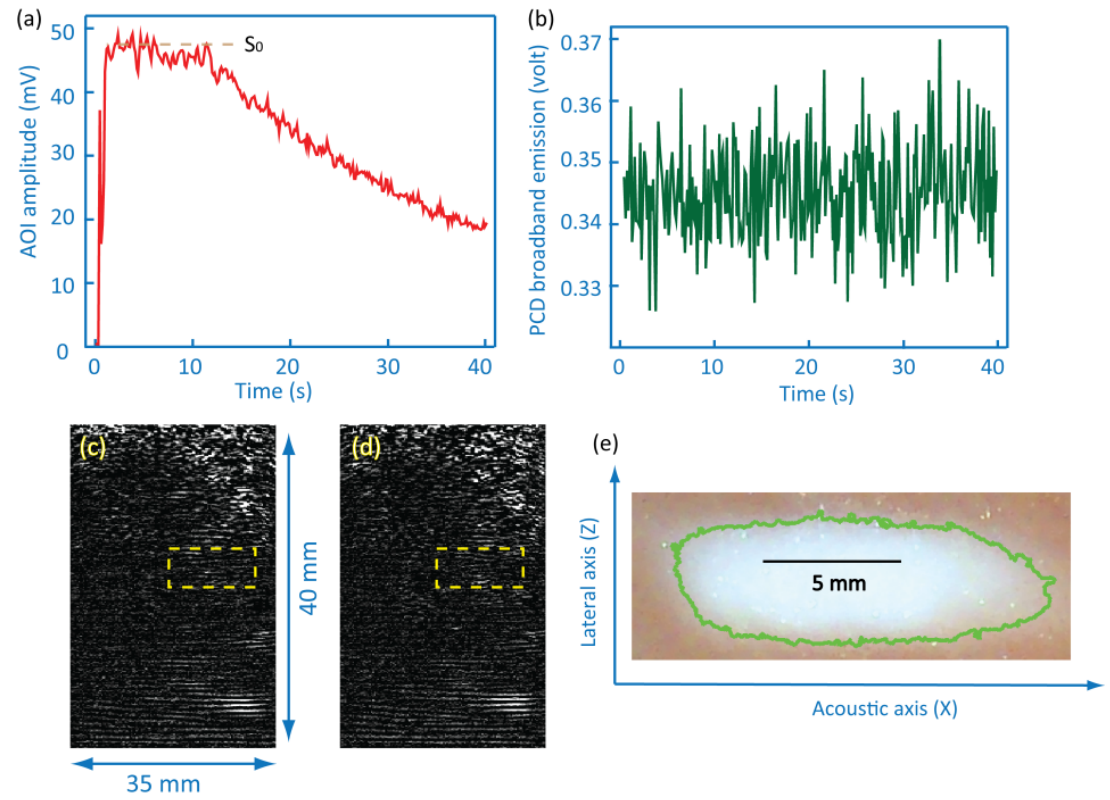

Fig. 4 (a) AOI magnitude output and (b) PCD signals as a function of exposure time; B-mode ultrasound images acquired immediately before (c) and after (d) the exposure (the dashed box indicates the approximate location of HIFU focus); (e) The lesion cross-section photograph generated by this exposure ( $\mathrm{X}$ is the HIFU axis from left to right).

Fig. 5(a) shows the AOI amplitude change $\Delta S$ as a function of ensuing lesion volume for exposures of two groups, with each measurement represented by one green square (Group 1) or one red circle (Group 2). No inertial cavitation or boiling was detected during any of these exposures. Even with fixed pressure and duration (Group 1), the ensuing lesions 
have a broad distribution of volumes ranging from 0 to $205 \mathrm{~mm}^{3}$. This is because the extent of a lesion is influenced by several factors in addition to pressure and duration, including the physical properties of the target and heating rates as well as the functional status of the overlying tissues between the transducer and the focal region ${ }^{33}$. In addition, some of the physical properties are temperature and time dependent. These factors make it difficult to predict the in situ heating in the focal region and the resulting shape and size of the ensuing lesion. When different pressures and durations are used, the lesion volumes exhibit large variations, although as a whole larger pressures and/or longer durations do tend to result in larger lesions (Fig. 5(b)). It is interesting to note, however, that the AOI amplitude is inversely proportional to the resultant lesion volume, and such correlation is not affected by the exact exposure parameters at the given range. This suggests that the evolution of AOI response as a function of exposure time can potentially be used to determine the onset of formation and the resulting volume of a lesion in real time.
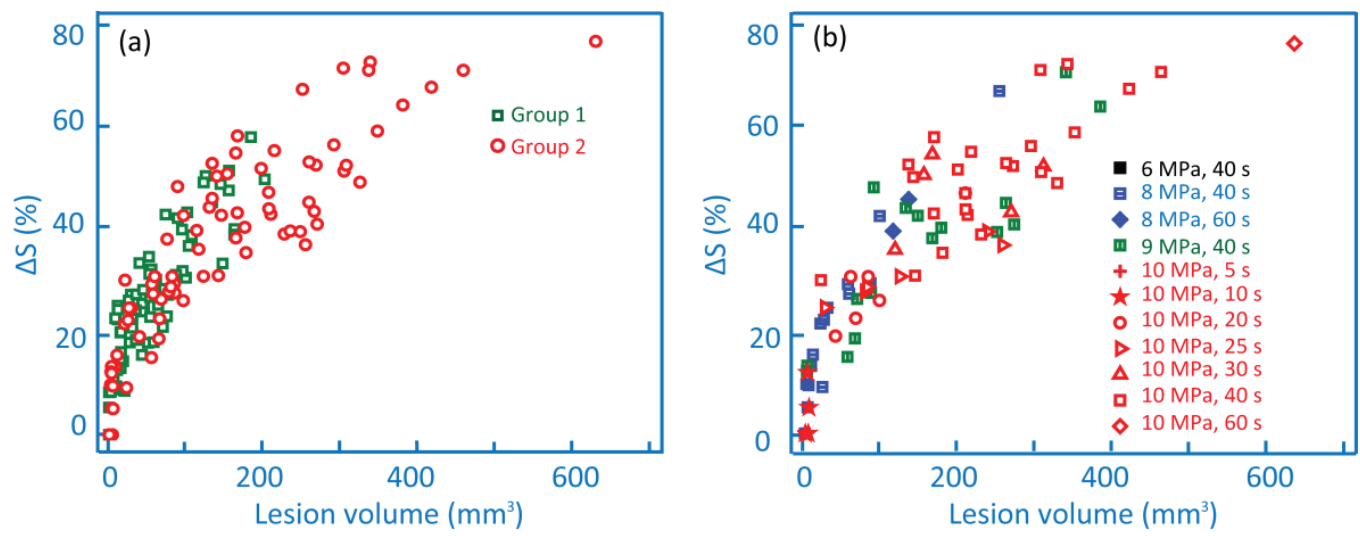

Fig. 5 (a) The AOI amplitude change $\Delta S$ as a function of ensuing lesion volume for Group 1 (green squares) and 2 (red circles) exposures; and (b) the correlation between AOI amplitude change and lesion volume of Group 2, sorted according to the exposure parameters (i.e. pressure and duration)

For HIFU treatments, it is very important to ensure that the desired target volume is necrosed while neighboring healthy tissues are not adversely affected. Therefore, predictable and repeatable shapes and sizes of necrosed regions are required to avoid inadequate or excessive heating. From this perspective, the AOI response is proposed as the criteria to control the exposure durations. Here we consider a fixed ultrasound pressure of $10 \mathrm{MPa}$ (Group 3, Table 1) and the exposure time is limited to produce a preset reduction in the AOI response $\Delta S_{p}$. When $\Delta S_{p}$ is reached, the HIFU exposure is terminated and the resulting lesion size determined. The aim is to determine whether or not the AO response can be used as a predictor for lesion size.

Figure 6 shows the statistical results of the resulting lesion volumes as a function the mean value of actual exposure durations to reach the preset $\Delta S_{p}$ at $20 \%, 30 \%, 40 \%, 50 \%$ and $60 \%$ respectively from left to right. The measurements are compared with exposures with a number of preset exposure durations at 25s, 30s, 40s, 50s, $60 \mathrm{~s}$ and $75 \mathrm{~s}$ (Group 4, Table 1). Note once again, there is no inertial cavitation or boiling detected for any of the measurements here. With comparable mean exposure duration, a 30 40\% decrease in the standard deviation of the ensuing lesion volumes can be obtained if using a preset AOI threshold to guide the exposure. This could potentially improve the efficiency and accuracy of treatment planning and evaluation. 


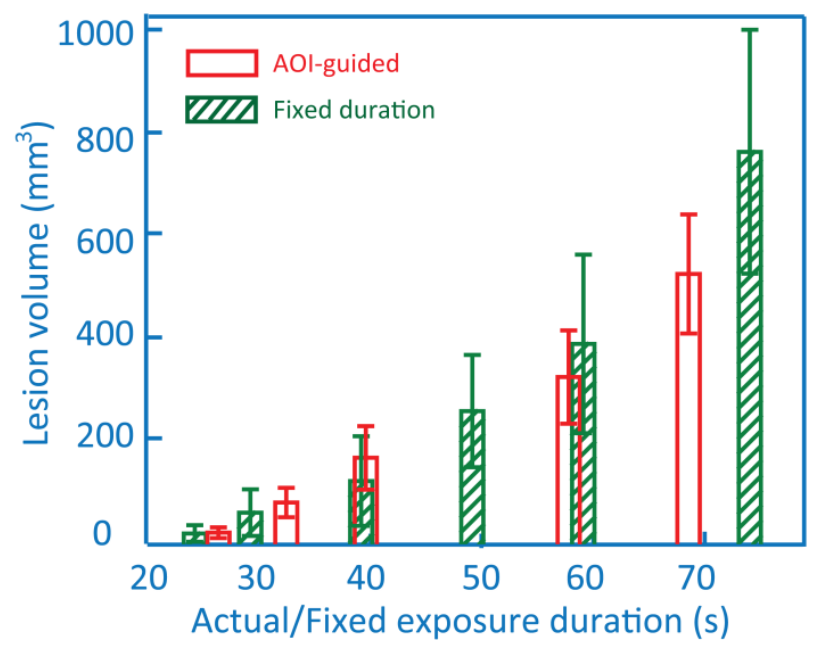

Fig. 6 Lesion volume distribution for exposures guided by AOI response (red, blank columns, with 20\%, 30\%, 40\%, 50\% and 60\% of $\Delta S_{p}$ from left to right) and exposures with fixed durations (green, filled columns, with $25 \mathrm{~s}, 30 \mathrm{~s}, 40 \mathrm{~s}, 50 \mathrm{~s}, 60 \mathrm{~s}$ and $75 \mathrm{~s}$ from left to right). The height of the column and the error bar represent the mean value and standard deviation of the lesion volumes respectively.

\section{CONCLUSIONS}

In summary an integrated acousto-optic imaging system was proposed to monitor and guide the HIFU treatments in the absence of inertial cavitation and boiling. It was shown that the AOI signal reduction for an exposure in ex vivo chicken breast tissue is correlated with the ensuing the lesion size. The system may potentially provide a real-time modality that can determine the onset and the ensuing volumes of necrosed regions, and to guide exposures during treatment. With recent advances in acousto-optic imaging ${ }^{34,35}$, it may be possible to extend this approach to in vivo HIFU treatment trials as well as other thermal ablations in optically accessible tissues in the near future.

\section{ACKNOWLEDGEMENT}

This work was supported by the Bernard M. Gordon Center for Subsurface Sensing and Imaging Systems (CENSSIS) under the Engineering Research Centers Program of the National Science Foundation (Award number EEC-9986821).

\section{REFERENCES}

1. J. G. Lynn, R. L. Zwemer, A. J. Chick, and A. E. Millar, "A new method for the generation and use of focused ultrasound in experimental biology," J Gen Physiol 26, 179-193 (1942).

2. W. J. Fry, W. H. Mosberg, J. W. Barnard, and F. J. Fry, "Production of focal destructive lesions in the central nervous system with ultrasound," J Neurosurg 11, 471-478 (1954).

3. G. R. ter Haar, D. Sinnett, and I. Rivens, "High intensity focused ultrasound - a surgical technique for treatment of discrete liver tumors," Phys Med Biol 34, 1743-1750 (1989).

4. G. Vallancien, E. Chartier-Kastler, and M. Harouni, "Focused extracorporeal pyrotherapy: experimental study and feasibility in man," Semin Urol 11, 7-9 (1993).

5. A. Gelet, J. Y. Chapelon, R. Bouvier, O. Rouviere, Y. Lasne, D. Lyonnet, and J. M. Dubernard, "Transrectal high-intensity focused ultrasound: minimally invasive therapy of localized prostate cancer," J Endourol 14, 519-528 
(2000).

6. A. H. Chan, V. Y. Fujimoto, D. E. Moore, R. W. Martin, and S. Vaezy, "An image-guided high intensity focused ultrasound device for uterine fibroids treatment," Med. Phys. 29, 2611-2620 (2002).

7. F. Wu, Z. Wang, Y. Cao, W. Chen, J. Bai, J. Zou, and H. Zhu, "A randomized clinical trial of high-intensity focused ultrasound ablation for the treatment of patients with localized breast cancer," Br J. Cancer 89, 2227-2233 (2003).

8. F. Wu, Z. Wang, W. Chen, J. Zou, J. Bai, H. Zhu, K. Li, F. Xie, C. Jin, H. Su, and G. Gao, "Extracorporeal focused ultrasound surgery for treatment of human solid carcinomas: early Chinese clinical experience " Ultrasound Med Biol 30, 245-260 (2004).

9. F. Wu, Z. Wang, and H. Zhu, "Feasibility of US-guided high-intensity focused ultrasound treatment in patients with advanced pancreatic cancer: initial experience," Radiology 236, 1034-1040 (2005).

10. T. J. Dubinsky, C. Cuevas, M. K. Dighe, O. Kolokythas, and J. H. Hwang, "High-intensity focused ultrasound: current potential and oncologic applications," AJR 190, 131-199 (2008).

11. I. Rivens, A. Shaw, J. Civale, and H. Morris, "Treatment monitoring and thermometry for therapeutic focused ultrasound," Int. J. Hyperthemia 23, 121-139 (2007).

12. K. Hynynen, W. R. Freund, H. E. Cline, C. A. H., R. D. Watkins, J. P. Vetro, and F. A. Jolesz, "A clinical, noninvasive, MR imaging-monitored ultrasound surgery method," Radiographics 16, 185-195 (1996).

13. C. Coussios, C. H. Farny, G. T. Haar, and R. A. Roy, "Role of acoustic cavitation in the delivery and monitoring of cancer treatment by high-intensity focused ultrasound (HIFU)," Int. J. Hyperthemia 23, 105-120 (2007).

14. I. F. Cilesiz, and A. J. Welch, "Light dosimetry: effects of dehydration and thermal damage on the optical properties of the human aorta," Appl. Opt. 32, 477-487 (1993).

15. A. M. K. Nilsson, C. Sturesson, D. L. Liu, and S. Andersson-Engels, "Changes in spectral shape of tissue optical properties in conjunction with laser-induced thermotherapy," Appl. Opt. 37, 1256-1267 (1998).

16. V. Quaresima, S. J. Matcher, and M. Ferrari, "Identification and quantification of intrinsic optical contrast for near-infrared mammography," Photochem. Photobiol. 67, 4-14 (1998).

17. A. N. Yaroslavsky, P. C. Schulze, I. V. Yaroslavsky, R. Schober, F. Ulrich, and H.-J. Schwartz, "Optical properties of selected native and coagulated human brain tissues in vitro in the visible and near infrared spectral range," Phys. Med. Biol. 47, 2059-2073 (2002).

18. T. D. Khokhlova, I. M. Pelivanov, O. A. Sapozhniko, V. S. Solomatin, and A. A. Karabutov, "Opto-acoustic diagnostics of the thermal action of high-intensity focused ultrasound on biological tissues: the possibility of its applications and model experiments," Quantum Electronics 36, 1097-1102 (2006).

19. M. Ben-David, R. Cantor, N. Balbul, M. Yehuda, and I. Gannot, "Measuring tissue heat penetration by scattered light measurements," Lasers in Surgery and Medicine 40, (2008).

20. M. Arsenault, M. Kolios, and W. M. Whelan, "Optoacoustic detection of thermal lesions," SPIE 7177, 71771V (2009).

21. X. Jin, Y. Xu, L. V. Wang, Y. R. Fang, C. I. Zanelli, and S. M. Howard, "Imaging of high-intensity focused ultrasound-induced lesions in soft biological tissue using thermoacoustic tomography," Med. Phys. 32, 5-11 (2005).

22. D. Dolfi, and F. Micheron, "Imaging process and system for transillumination with photon frequency marking," in International Patent WO 89/00278, (1989).

23. F. A. Marks, H. W. Tomlinson, and G. W. Brooksby, "A comprehensive approach to breast cancer detection using light: photon localization by ultrasound modulation and tissue characterization by spectral discrimination," SPIE $\mathbf{1 8 8 8}$, 
500-510 (1993).

24. L. V. Wang, S. L. Jacques, and X. Zhao, "Continuous-wave ultrasonic modulation of scattered laser light to image objects in turbid media," Opt. Lett. 20, 629-631 (1995).

25. W. Leutz, and G. Maret, "Ultrasonic modulation of multiply scattered light," Phyisca B 204, 14-19 (1995).

26. L. V. Wang, "Mechanisms of ultrasound modulation of multiply scattered coherent light: An analytic model," Phys. Rev. Lett. 87, 043903 (2001).

27. C. Kim, R. J. Zemp, and L. V. Wang, "Intense acoustic bursts as a signal-enhancement mechanism in ultrasound-modulated optical tomography," Opt. Lett. 31, 2423-2425 (2006).

28. T. W. Murray, L. Sui, G. Maguluri, R. A. Roy, A. Nieva, F. J. Blonigen, and C. A. DiMarzio, "Detection of ultrasound-modulated photons in diffuse media using the photorefractive effect," Opt. Lett. 29, 2509-2511 (2004).

29. P. Lai, R. A. Roy, and T. W. Murray, "Quantitative characterization of turbid medium using pressure contrast acousto-optic imaging," Optics Letters 34, 2850-2852 (2009).

30. P. Lai, R. A. Roy, and T. W. Murray, "Sensing the optical properties of diffusive media by acousto-optic pressure contrast imaging," SPIE 7177, 71771G (2009).

31. C. H. Farny, R. G. Holt, and R. A. Roy, "Temporal and spatial dependence of HIFU-induced inertial and hot-vapor cavitation with a diagnostic ultrasound system," Ultrasound Med Biol 35, 603-615 (2009).

32. J. R. McLaughlan, "An investigation into the use of cavitation for the optimisation of high intensity focused ultrasound (HIFU) treatments," in Joint Department of Physics, Institute of Cancer Research and Royal Marsden NHS Trust, (University of London, London, 2008).

33. Z. Wang, J. Bai, F. Li, Y. Du, S. Wen, K. Hu, G. Xu, P. Ma, N. Yin, W. Chen, F. Wu, and R. Feng, "Study of a "biological focal region" of high-intensity focused ultrasound," Ultrasound Med Biol 29, 749-754 (2003).

34. G. Rousseau, A. Blouin, and J.-P. Monchalin, "Ultrasound-modulated optical imaging using a powerful long pulse laser," Opt. Express 16, 12577-12590 (2008).

35. Y. Li, P. Hemmer, C. Kim, H. Zhang, and L. V. Wang, "Detection of ultrasound-modulated diffuse photons using spectral hole buring," Optics Express 16, 14862-14874 (2008). 